\title{
Are you stressed?
}

\author{
C. Rischpler, $M D,{ }^{\mathrm{a}} M$. Totzeck, $M D^{\mathrm{b}}$ \\ a Department of Nuclear Medicine, University Hospital Essen, University of Duisburg-Essen, \\ Essen, Germany \\ b Department of Cardiology and Vascular Medicine, University Hospital Essen, West German \\ Heart and Vascular Center, University of Duisburg, Essen, Germany
}

Received May 22, 2018; accepted May 22, 2018

doi: $10.1007 /$ s $12350-018-1332-6$

\section{See related article, pp. 1888-1897}

Myocardial perfusion imaging is still a mainstay for the diagnosis and management of coronary artery disease. As a consequence, millions of US patients are examined with this technique every year. ${ }^{1}$ There are crucial points regarding the conduction of the procedure in order to guarantee optimal patient preparation, image quality, and analysis. Among those critical points are the choice and dose of the radiotracer, ECG-gated testing (for the assessment of left ventricular volumes and ejection fraction) and the compulsory quantification of the extent of affected myocardium (scar vs. ischemia, percentage of the left ventricular myocardium). (More details on state-of-the-art myocardial perfusion imaging can be found in the respective guidelines as released, e.g., by ASNC, SNMMI, or EANM). ${ }^{2}$ Another utterly important point regarding the conduction of myocardial perfusion imaging tests is that patients are recommended to be stressed adequately. ${ }^{3}$ Whenever possible, physical exercise using either a treadmill or a bicycle ergometer should be the preferred method. If the patient is unable to exercise adequately, pharmacological agents to increase myocardial blood flow sufficiently to detect hemodynamically relevant coronary stenoses can be applied with comparable sensitivity and specificity. ${ }^{4,5}$ Currently, the most frequently used pharmacological stressors are regadenoson and adenosine. These substances bind to the adenosine receptor A2A in coronary arteries and initiate a dilatation of these vessels, which,

Reprint requests: C. Rischpler, MD, Department of Nuclear Medicine, University Hospital Essen, University of Duisburg-Essen, Essen, Germany; rischpler@gmail.com

J Nucl Cardiol 2019;26:1898-900.

1071-3581/\$34.00

Copyright (C) 2018 American Society of Nuclear Cardiology. in turn, increases myocardial blood flow up to fourfold. Dipyridamole acts via the same axis by an increase of intrinsic adenosine (through the inhibition of the cellular reuptake of adenosine into platelets, red blood cells, and endothelial cells). Unfortunately, all of these pharmacological stressors may have considerable side effects: Adenosine also binds to other adenosine receptor subtypes, which may result in significant arrhythmias (e.g., AV block and other arrhythmias), dilatation of peripheral vessels, hypotension, reflex tachycardia, and bronchoconstriction. Regadenoson is thought to have less side effects as it binds to the other adenosine receptor subtypes with lower affinity. The only infrequently used pharmacological stressor in myocardial perfusion imaging is dobutamine. Dobutamine is a sympathomimetic drug and is used in patients with severe COPD or asthma as it does not cause bronchoconstriction.

It is known that without adequate exercise the sensitivity of myocardial perfusion studies decreases significantly. ${ }^{3}$ Accordingly, patients that are scheduled to undergo exercise for myocardial perfusion imaging are asked to stop taking medicine that may interfere with an increase of the heart frequency, particularly betablockers. For pharmacological vasodilation using adenosine, regadenoson or dipyridamole patients are asked to abstain from any methylxanthines (such as theophylline, caffeine, and chocolate) as they effectively block the adenosine receptor. ${ }^{6}$ This may result in a falsenegative scan or, at least, decrease the extent of the stress-induced perfusion defect. Rates of false-negative myocardial perfusion imaging studies have been reported to be as high as 5 to $10 \%{ }^{7,8}$ Data suggest that up to one third of these false-negative scans are related to inadequate stress either through administration errors or drug interactions. ${ }^{9}$ This points out the importance of adequate pharmacological stress and that it is crucial to ask patients undergoing myocardial perfusion imaging to abstain from methylxanthines. Interestingly, caffeine 
was still present in the blood in $40 \%$ of the patients when abstaining from caffeine for 24 hours. ${ }^{10}$ This indicates, that despite adherence to current guidelines some patients may have substantial caffeine blood levels which may interfere with myocardial perfusion imaging. Another problem is the lack of any definite clinical sign to conclude that patients were adequately stressed following the injection of adenosine receptor agonists. ${ }^{11}$ An inadequate hemodynamic response is even known be an independent prognostic predictor of poor outcome. ${ }^{12}$ Accordingly, an independent, easily accessible and noninvasive marker for the estimation of hemodynamic response to adenosine receptor agonists would be desirable and of high clinical relevance. Just recently, the so-called splenic switch-off sign has been described as a tool for the assessment of stress adequacy in adenosine myocardial perfusion imaging. ${ }^{13}$ This phenomenon occurs as adenosine receptor A1-stimulation reduces splanchnic artery perfusion. This was first noted on MR perfusion imaging in terms of a visual attenuation of splenic perfusion during adenosine application. $^{14}$ Initial studies suggest that the splenic switch-off sign is a more reliable marker of hemodynamic response to adenosine application than changes in heart rate, blood pressure or the occurrence of physical symptoms. ${ }^{15}$ The problem that the switch-off sign can only be seen following contrast media application has been overcome by using a native T1-mapping technique for the quantification of tissue blood volumes without contrast application. ${ }^{16}$ The splenic reaction to adenosine stress may thus be used to monitor and guide the dose of adenosine application. In one study, 74 of 955 patients did not respond to standard adenosine administration (140 $\mathrm{mg} / \mathrm{kg} / \mathrm{min})$. Concomitantly, adenosine infusion dose was increased up to $210 \mathrm{mg} / \mathrm{kg} / \mathrm{min}$. 61 of the 74 patients $(82 \%)$ also demonstrated a splenic switch-off. ${ }^{17}$ To overcome the dependence on spleen perfusion MRI to monitor an adequate response to adenosine, ultrasound may be used. A recent study compared the onset of splenic switch-off on MRI with a decrease of splenic artery peak velocity using color Doppler in 26 patients. 24 patients with splenic switch-off demonstrated a drop in splenic artery peak velocity whereas the failing splenic switch-off in the remaining 2 patients was paralleled by an absent drop in splenic artery peak velocity on ultrasound. ${ }^{18}$

In the current issue of the Journal of Nuclear Cardiology, Bami et al report for the first time in a very nice study on the utility of the splenic response under dipyridamole stress in Rubidium-82 PET myocardial perfusion imaging. ${ }^{19}$ The authors suggest an simplified approach to estimate the splenic response and establish a cutoff value for a normal splenic response using a cohort of patients with normal global myocardial flow reserve and normal PET interpretation. Also, the authors followed up 703 patients with normal PET myocardial perfusion scans regarding the occurrence of major adverse cardiac events (MACE) within 1 year. Patients with normal summed stress scores (SSS $<4$ ) and normal summed difference scores $($ SDS $<2)$ had a significantly higher MACE rate in case they were splenic nonresponders $(7.8 \%$ vs $2.9 \%$ and $7.4 \%$ vs $2.2 \%$, respectively). Most importantly, an absent splenic response remained an independent prognostic factor in a multivariate analysis. Even though, these results are highly promising, there are two factors that may prevent the broad application of this approach in clinical routine. First, a recent MRI study has demonstrated that the splenic switch-off phenomenon may be less sensitive to caffeine intake than the vasodilation in coronary arteries. ${ }^{20}$ Therefore, the splenic response to adenosine may not be used as a marker of adequate hemodynamic response. Secondly - this may be the death knell for the splenic switch-off sign as a novel, important prognostic marker - this phenomenon can only be observed when patients are stressed with adenosine or dipyridamole, but not with regadenoson or dobutamine. Since the approval of regadenoson by the FDA for the use as a pharmacological vasodilator for myocardial perfusion imaging in 2008, this agent is now by far the most often used stress agent in nuclear medicine laboratories in the US, covering about $84 \%$ of the market by $2013 .^{21} \mathrm{We}$ are thus still left with the question: are you stressed (adequately)?

\section{Disclosure}

The authors have nothing to disclose.

\section{References}

1. 2008 Nuclear Medicine: Market Summary Report. Des Plains: IMV Medical Information Division; 2008. p. IV19-30.

2. Henzlova MJ, Duvall WL, Einstein AJ, Travin MI, Verberne HJ. ASNC imaging guidelines for SPECT nuclear cardiology procedures: Stress, protocols, and tracers. J Nucl Cardiol. 2016;23:60639.

3. Iskandrian AS, Heo J, Kong B, Lyons E. Effect of exercise level on the ability of thallium-201 tomographic imaging in detecting coronary artery disease: analysis of 461 patients. J Am Coll Cardiol. 1989;14:1477-86.

4. Gupta NC, Esterbrooks DJ, Hilleman DE, Mohiuddin SM. Comparison of adenosine and exercise thallium-201 single-photon emission computed tomography (SPECT) myocardial perfusion imaging. The GE SPECT Multicenter Adenosine Study Group. J Am Coll Cardiol. 1992;19:248-57.

5. Nishimura S, Mahmarian JJ, Boyce TM, Verani MS. Equivalence between adenosine and exercise thallium-201 myocardial tomography: A multicenter, prospective, crossover trial. J Am Coll Cardiol. 1992;20:265-75. 
6. Tejani FH, Thompson RC, Kristy R, Bukofzer S. Effect of caffeine on SPECT myocardial perfusion imaging during regadenoson pharmacologic stress: A prospective, randomized, multicenter study. Int J Cardiovasc Imaging. 2014;30:979-89.

7. Go RT, Marwick TH, MacIntyre WJ, Saha GB, Neumann DR, Underwood DA, et al. A prospective comparison of rubidium-82 PET and thallium-201 SPECT myocardial perfusion imaging utilizing a single dipyridamole stress in the diagnosis of coronary artery disease. J Nucl Med. 1990;31:1899-905.

8. Mc Ardle BA, Dowsley TF, deKemp RA, Wells GA, Beanlands RS. Does rubidium-82 PET have superior accuracy to SPECT perfusion imaging for the diagnosis of obstructive coronary disease?: A systematic review and meta-analysis. J Am Coll Cardiol. 2012;60:1828-37.

9. Kidambi A, Sourbron S, Maredia N, Motwani M, Brown JM, Nixon J, et al. Factors associated with false-negative cardiovascular magnetic resonance perfusion studies: A clinical evaluation of magnetic resonance imaging in coronary artery disease (CEMARC) substudy. J Magn Reson Imaging. 2016;43:566-73.

10. Jacobson AF, Cerqueira MD, Raisys V, Shattuc S. Serum caffeine levels after 24 hours of caffeine abstention: Observations on clinical patients undergoing myocardial perfusion imaging with dipyridamole or adenosine. Eur J Nucl Med. 1994;21:23-6.

11. Mishra RK, Dorbala S, Logsetty G, Hassan A, Heinonen T, Schelbert HR, et al. Quantitative relation between hemodynamic changes during intravenous adenosine infusion and the magnitude of coronary hyperemia: implications for myocardial perfusion imaging. J Am Coll Cardiol. 2005;45:553-8.

12. Hage FG, Dean P, Iqbal F, Heo J, Iskandrian AE. A blunted heart rate response to regadenoson is an independent prognostic indicator in patients undergoing myocardial perfusion imaging. J Nucl Cardiol. 2011;18:1086-94.
13. Manisty C, Ripley DP, Herrey AS, Captur G, Wong TC, Petersen SE, et al. Splenic switch-off: A tool to assess stress adequacy in adenosine perfusion cardiac MR imaging. Radiology. 2015;276:732-40.

14. Morato M, Sousa T, Albino-Teixeira A. Purinergic receptors in the splanchnic circulation. Purinergic Signal. 2008;4:267-85.

15. Hosking A, Koulouroudias M, Zemrak F, Moon JC, Rossi A, Lee A, et al. Evaluation of splenic switch off in a tertiary imaging centre: Validation and assessment of utility. Eur Heart J Cardiovasc Imaging. 2017;18:1216-21.

16. Liu A, Wijesurendra RS, Ariga R, Mahmod M, Levelt E, Greiser A, et al. Splenic T1-mapping: A novel quantitative method for assessing adenosine stress adequacy for cardiovascular magnetic resonance. J Cardiovasc Magn Reson. 2017;19:1.

17. Walkden M, Bryant J, Abbas A, Harden S, Shambrook J, Peebles C. Turning up the adenosine turns off the spleen. J Cardiovasc Magn Reson. 2015;17(Suppl 1):T1.

18. Klein-Wiele O, Sherifa W, Garmer M, Kara K, Gronemeyer D, Hailer B. Assessment of systemic adenosine effect using color doppler ultrasound of the splenic artery-feasibility and potential clinical utility for coronary interventions. Ultrasound Med Biol. 2018;44:1119-23.

19. Bami K, Tewari S, Guirguis F, Garrard L, Guo A, Hossain A, et al. Prognostic utility of splenic response ratio in dipyridamole PET myocardial perfusion imaging. J Nucl Cardiol. 2018. https://doi. org/10.1007/s12350-018-1269-9.

20. Kuijpers D, van Dijk R, van Assen M, Kaandorp TAM, van Dijkman PRM, Vliegenthart R, et al. Disagreement between splenic switch-off and myocardial T1-mapping after caffeine intake. Int J Cardiovasc Imaging. 2018;34:625-32.

21. American Society of Nuclear Cardiology/MedAxiom Nuclear Survey 2013. J Nucl Cardiol 2014;21:5-88. 